\title{
FREEZE-FRACTURE STUDIES ON BARLEY PLASTID MEMBRANES IV. ANALYSIS OF FREEZE-FRACTURE PARTICLE SIZE AND SHAPE
}

\author{
by \\ DAVID J. SIMPSON \\ Department of Physiology, Carlsberg Laboratory, \\ Gamle Carlsbergvej 10, DK-2500 Copenhagen Valby
}

Keywords: chlorina-f2, plastic deformation, rotary shadowing, statistical analysis

The shape and size of freeze-fracture particles of rotary shadowed barley thylakoids were measured and plotted as three-dimensional histograms. It was not possible to resolve discrete sub-populations of particles, distinct in shape or size, on any of the fracture faces examined. It is concluded that much of the variation in particle shape and size was due to plastic deformation during freeze-fracturing, and to shadowing artefacts. The suggestion is made that EFs particles are uniformly sized in vivo. Particle size measurements can be useful, however, in making comparisons between different fracture faces and between corresponding faces of wild-type and mutant membranes, as long as the limitations of the technique are considered. The differences between corresponding faces of wild-type and chlorina- $f 2$ thylakoids were found to be statistically significant for the EFs. ESs and PF faces.

\section{INTRODUCTION}

The polypeptide components of biological membranes make specific associations with one another in defined stoichiometry, forming functional units. Such functional units are likely to correspond to the structural entities revealed as individual particles within the membrane by freeze-fracturing. This concept is supported by the successful isolation of multi-polypeptide complexes from various membranes, with the aid of mild detergent treatment, and the subsequent incorporation of these complexes into lipid bilayer vesicles with the concomitant appearance of freeze-fracture particles $(3,10,17$, 18).

In connection with these studies, it would be

Abbreviations: $\mathrm{EFs}=$ endoplasmic fracture (stacked), $\mathrm{EFu}=$ endoplasmic fracture (unstacked), $\mathrm{ES}=$ endoplasmic surface (destacked), LHC = light-harvesting chlorophyll-protein complex, PFs = protoplasmic face (stacked), $\mathrm{PFu}=$ protoplasmic face (unstacked). ESs = endoplasmic surface (stacked). 
useful to know the precise size of these freezefracture particles in order to calculate their molecular weight, and to correlate this with their polypeptide composition (see 6). Since most studies have employed uni-directionally shadowed replicas, it has only been possible to obtain an average value for particle size from their size distribution, and it has been assumed that these particles have a circular cross-section at the freeze-fracture plane (2). This was shown not to be the case for the EFs particles of the thylakoid membrane from barley chloroplasts which have been induced to form regular arrays of particles (19).

It is now possible to make direct measurements of particle shape and size from rotary shadowed freeze-fracture replicas (11). This was used in a recent paper (20) to compare the size of particles from corresponding faces of thylakoids of wild-type and a mutant of barley. The present paper examines the distribution of particle size and shape for the thylakoids of wild-type and the chlorina- $f 2$ mutant using rotary shadowed freeze-fracture replicas. It has been reported that up to four discrete size classes can be detected from particle size histograms of the EFs face of uni-directionally shadowed freeze-fractured thylakoids $(2,7,8)$.

It is thought that all the particles of the inner thylakoid surface (ESs) represent projections of the EFs particles $(12,19,23)$. The thylakoid membrane therefore offers the opportunity to compare the size and shape of particles which have been subjected to freeze-fracturing (EFs particles) with equivalent particles revealed by freeze-etching (ESs particles).

\section{MATERIALS AND METHODS}

The data analysed in this paper were obtained from rotary shadowed freeze-fracture replicas described in SIMPSON (1979). Measurements of the large and small axes of 500 particles from each face were sorted and placed into size classes, each class being $10 \AA$ square. The particle frequency was plotted against the dimensions of the large and small axes in the form of a threedimensional histogram, using computer programs developed by JøRGen PEETz.

The statistical analysis of these histograms from corresponding faces of wild-type and chlorina- $f 2$ barley thylakoids was performed by SteEn LaRSEN, using the $\chi^{2}$-test. The $\chi^{2}$ value is given by:

$$
\begin{aligned}
& \mathcal{X}^{2}=\sum_{j} \frac{\left(x_{1 j}-1 / 2\left(x_{1 j}+x_{2 j}\right)\right)^{2}}{1 / 2\left(x_{1 j}+x_{2 j}\right)} \\
& \text { where } \quad x_{1 j}= \text { value for wild-type } \\
& x_{2 j}= \text { value for mutant in } \\
& \text { corresponding class. }
\end{aligned}
$$

Comparisons were made only between corresponding classes which contained at least 5 particles.

\section{RESULTS}

The distributions of particle size and shape for the different fracture faces of wild-type and chlorina- $f 2$ thylakoids are shown as threedimensional histogram plots in Figures 1 and 4. Figure 3 gives the size distribution of particles revealed by freeze-etching on the endoplasmic surface of stacked thylakoids (ESs) and in arrays on the endoplasmic surface of destacked thylakoids (ES). The latter are compared with freezefracture particles in liposomes containing the light-harvesting chlorophyll $a / b$-protein 2 . In agreement with particle size histograms of unidirectionally shadowed replicas (19), there was a wide spread of sizes, particularly for the large axis, and especially for the EFs face (Figures 1a, b). The majority of particles in all diagrams lay within an area defined by the equations:

$$
\begin{aligned}
& x \geqslant y \quad \text { where } \quad x=\text { large axis } \\
& \text { and } x \leqslant 2 y \quad y=\text { small axis }
\end{aligned}
$$

Since the size of the small axis cannot be greater than the large axis, no particles fall to the left of the line $\mathrm{x}=\mathrm{y}$, and the fact that very few fell to the right of the line $\mathrm{x}=2 \mathrm{y}$ means that particle shape varies between circular and elliptical, with a maximum eccentricity of 2. Particles sizes appeared to be distributed about a single peak. There was no indication that particles on any face consisted of a number of discrete populations resolvable on the basis of size or shape.

Figure 1a shows the distribution of particle sizes for the EFs face of wild-type barley thylakoids. The size distribution is skewed towards the right of the graph, indicating that 

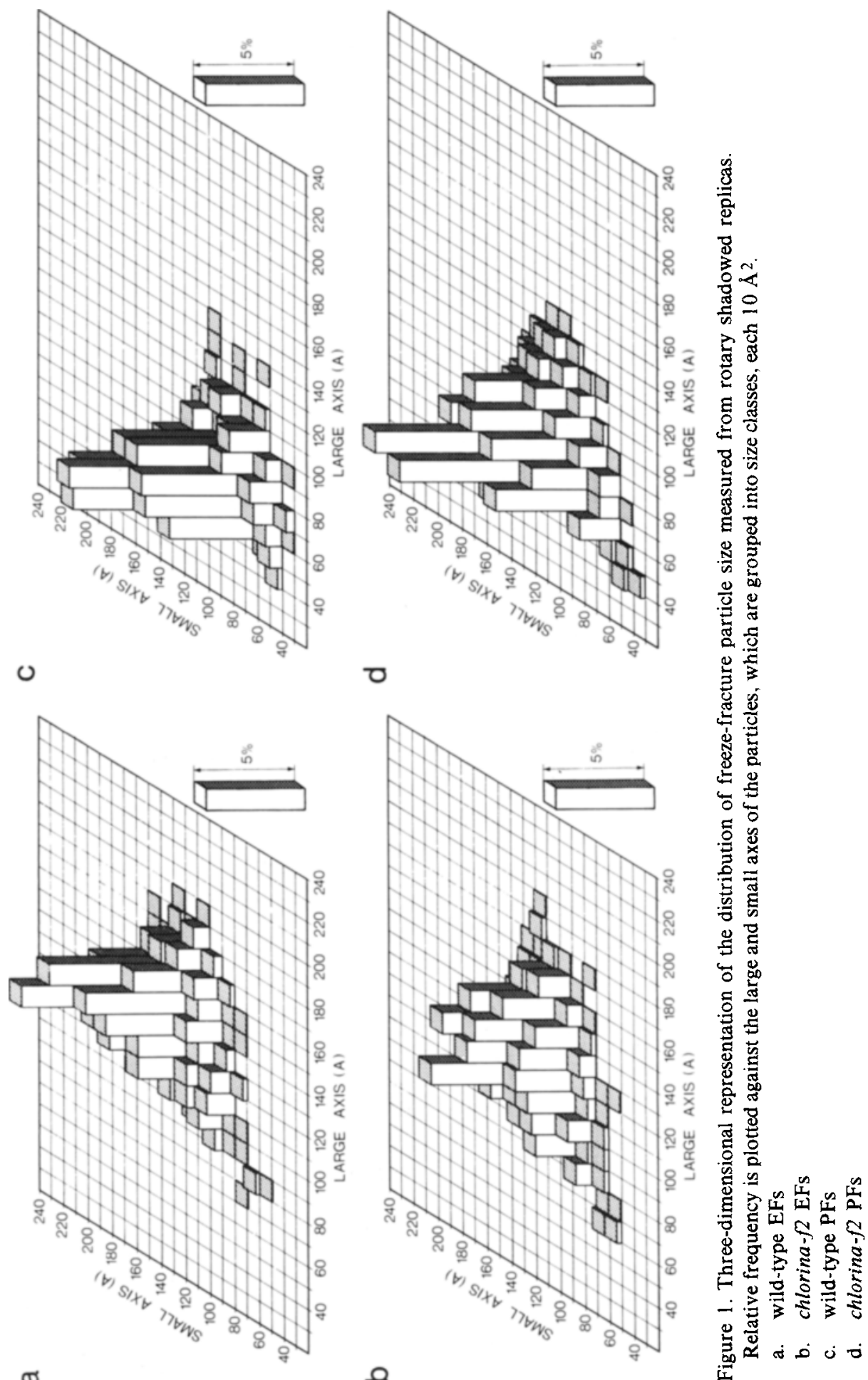

ช

م 

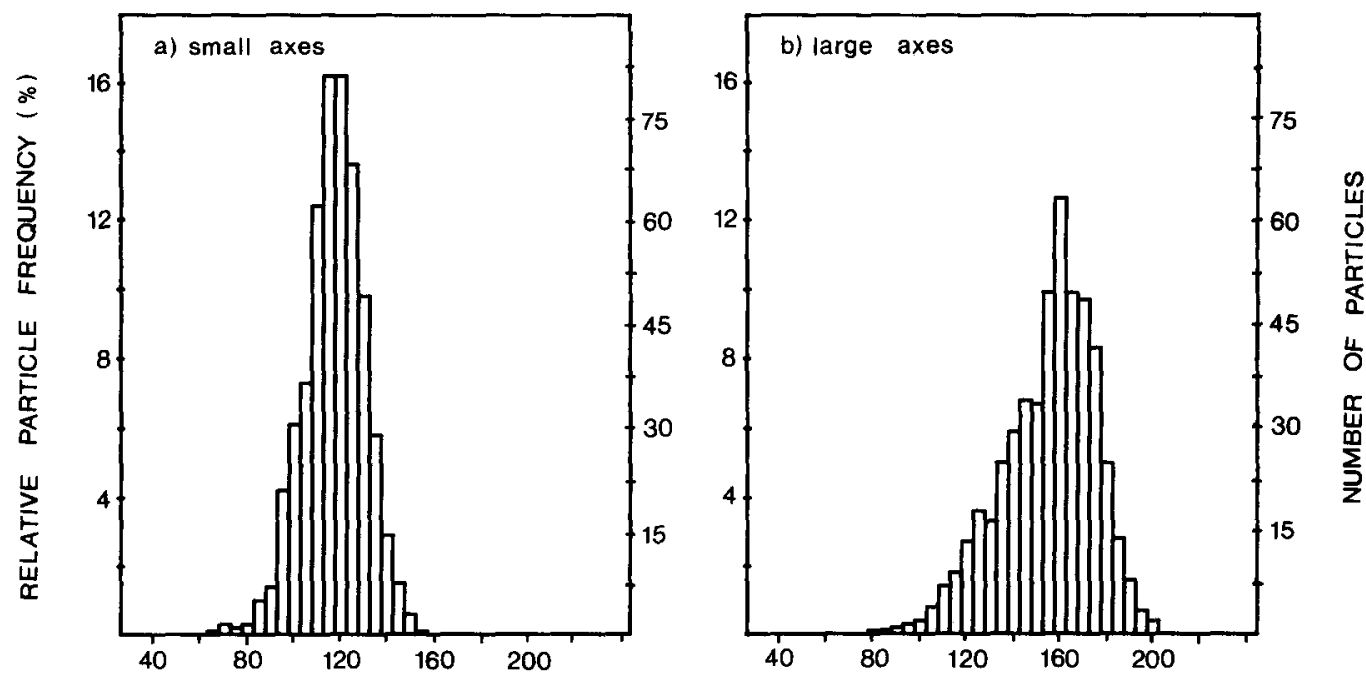

PARTICLE DIAMETER (A)

Figure 2. Two-dimensional histograms of the size distribution of the small and large axes of wild-type EFs particles (cf Fig. la).

The sizes of the small axes are normally distributed, while those of the large axes are skewed to the right, with no indication of discrete size classes.

most particles are large. This is also shown in the histogram of the particle large axis derived from the same data (Figure 2), whereas the small axis is normally distributed. The EFs particles from chlorina- $f 2$ thylakoids were proportionately smaller than wild-type and more uniformly distributed in size, with no tendency to cluster around more than a single mode (Figure $1 \mathrm{~b}$ ). The particles on the PFs face of wild-type (Figure 1c) were smaller than those of chlorina- $f 2$ (Figure 1d) and in both cases, PFs particles were less elliptical and smaller than those found on the EFs face from the same material.

The particles on the inner surface of the stacked regions of the thylakoid (ESs) revealed by freeze-etching, were also measured (Figures $3 \mathrm{a}, \mathrm{b})$. They were larger than the corresponding EFs face particles (Figures $1 \mathrm{a}, \mathrm{b}$ ) and consisted of a population distributed symmetrically about a single mode. Particles on the wild-type ESs were larger than those on the ESs of chlorina- $f 2$ to approximately the same degree that wild-type EFs particles were larger than chlorina- 2 EFs particles. These values are given in Table I. When the size of particles in ES arrays were measured and plotted (Figures 3c, d), the standard deviation of the distribution was smaller than unarrayed ESs particles.

Table I

Particle size and density data
(values are \pm standard error)

\begin{tabular}{llrr}
\hline & Face & wild-type & chlorina- $f 2$ \\
\hline Density & PFu $\S$ & $4553 \pm 25$ & $4092 \pm 25$ \\
$\left(\right.$ No. $\left./ \mu \mathrm{m}^{2}\right)$ & $\mathrm{EFu}$ & $347 \pm 6$ & $491 \pm 7$ \\
& $\mathrm{PFs}$ & $6257 \pm 33$ & $1124 \pm 35$ \\
& $\mathrm{EFs}$ & $1624 \pm 11$ & $2004 \pm 11$ \\
& $\mathrm{ESs}$ & $1450 \pm 15$ & $2054 \pm 17$ \\
\hline Size $(\AA)$ & $\mathrm{PFu}$ & $93 \times 112$ & $91 \times 109$ \\
& EFu & $105 \times 126$ & $104 \times 121$ \\
& $\mathrm{PFs}$ & $74 \times 93$ & $90 \times 103$ \\
& EFs & $117 \times 155$ & $104 \times 138$ \\
& ESs & $142 \times 191$ & $122 \times 174$ \\
& EF array & $102 \times 175$ & \\
& ES array & $130 \times 195$ & $115 \times 178$ \\
\hline
\end{tabular}

§ Data from SimPSON $(1978,1979)$ 

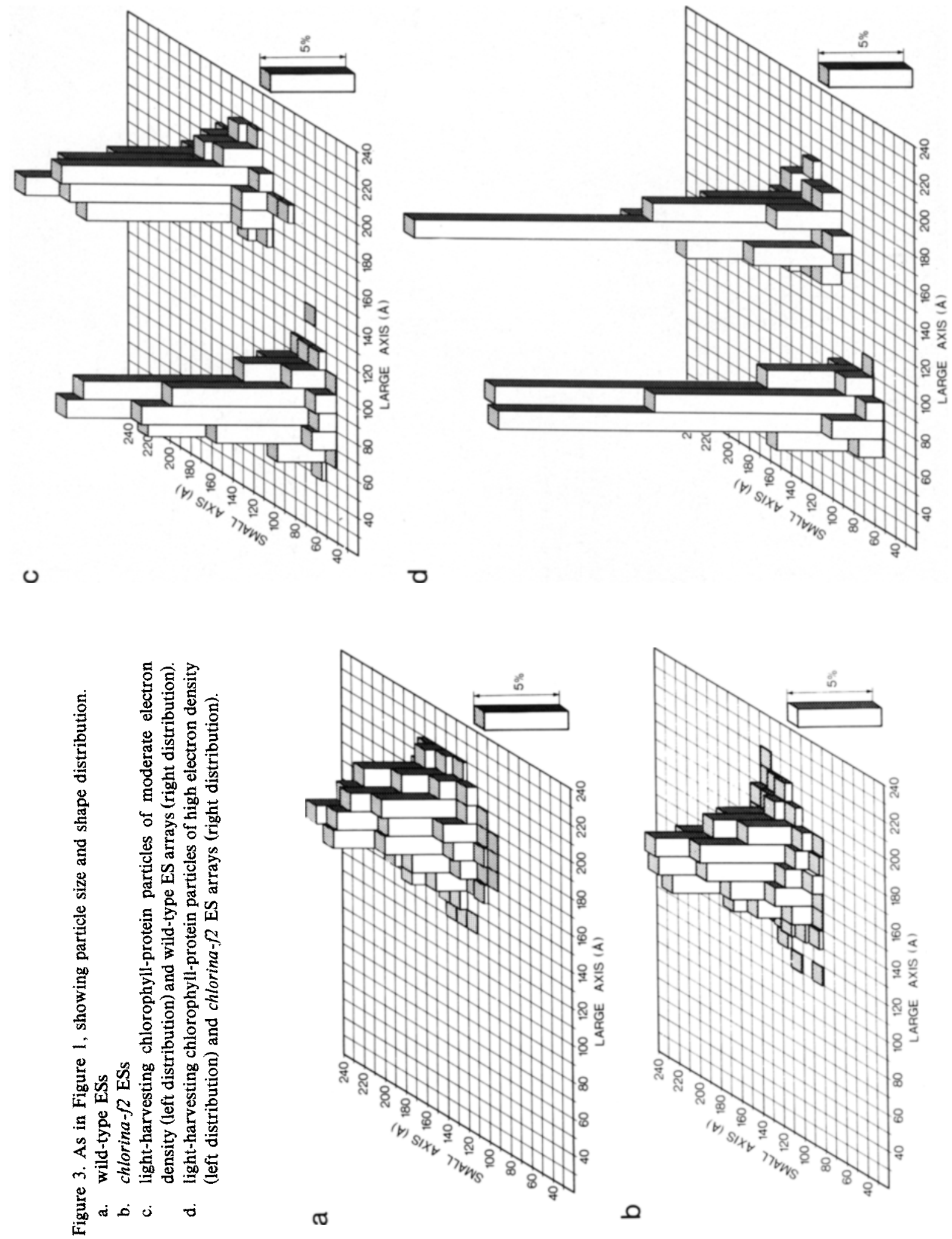

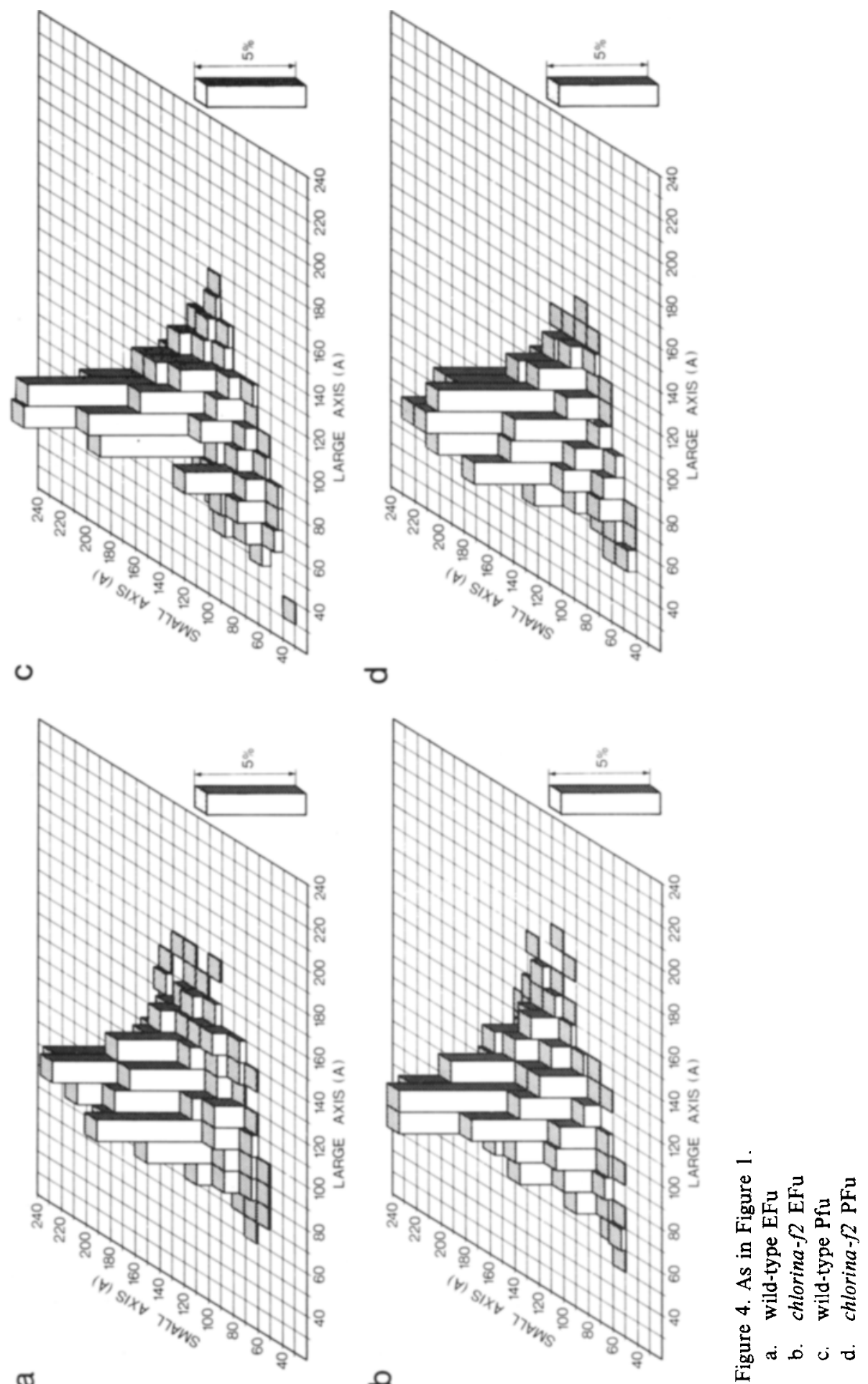
Table II

Statistical analysis of particle size data ( $d f=$ degrees of freedom)

\begin{tabular}{lccc}
\hline Face & $x^{2}$ value & df & Significance level \\
\hline PFs & 143.9 & 20 & $<0.0005$ \\
EFs & 143.3 & 28 & $<0.0005$ \\
ESs & 206.1 & 15 & $<0.0005$ \\
PFu & 33.4 & 28 & 0.2 \\
EFu & 23.9 & 28 & 0.7 \\
\hline
\end{tabular}

Liposomes containing the light-harvesting chlorophyll $a / b$-protein 2 revealed particles when freeze-fractured (Figure 5). Most of the particles were hexagonally packed with a centreto-centre spacing of $122 \AA(20)$, indicating that

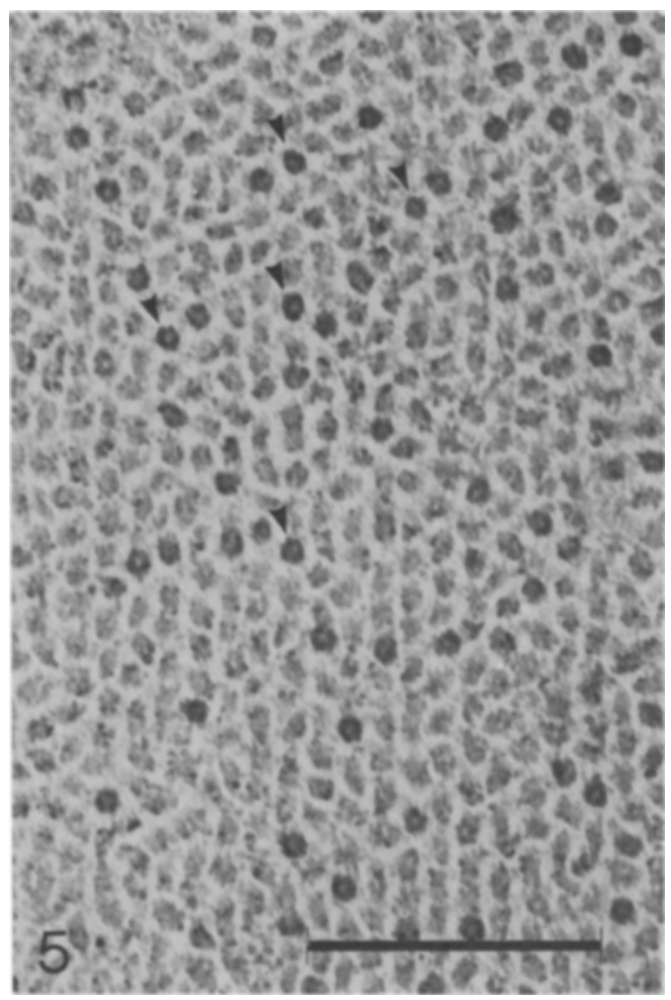

Figure 5. Rotary shadowed freeze-fracture replica of light-harvesting chlorophyll-protein in liposomes, demonstrating the presence of some particles with high electron density (arrowheads) among the majority of moderate electron density. $300,000 \times$. (Bar $=$ $0.1 \mu \mathrm{m})$. the particles were very similar, if not identical, in size and composition. The majority of such particles were of moderate electron density and their size distribution is shown in Figure 3c. It should be noted that although these particles were distributed over a narrower size range than those found on a thylakoid fracture face (Figures 1 and 4), there was still a significant variation in the measured size of an apparently uniformly sized population. This variation was similar to that found for particles in ES arrays (Figure 3c). A small number of particles in the liposomes were highly electron dense, with sharply defined edges and were found to be more uniform in size than the rest (Figure $3 \mathrm{~d}$ ) and less elliptical $(77.4 \AA \times 87.4 \AA$ versus $70.3 \AA \times 94.6 \AA)$.

The data for the EFu and PFu faces of wildtype and chlorina- $f 2$ thylakoids are shown in Figure 4. Visual examination of these histograms revealed only small differences in particle size distribution between corresponding faces of wild-type and mutant thylakoids. The data for mutant and wild-type were compared by statistical analysis using the $x^{2}$-test. Highly significant differences were found between the ESs, EFs and PFs of wild-type thylakoids and the corresponding faces of the chlorina- $f 2$ mutant (Table II).

\section{DISCUSSION}

Measurement of the particle size distribution of freeze-fracture faces has been used as a means of objectively characterising electron micrographs of such faces $(8,22)$. Apart from yielding a value for the average size of the particles, particle size histograms have been used to demonstrate the existence of discrete sub-populations of particles which differ in size $(2,7,23)$. Furthermore, changes in the relative proportions of these sub-populations on the EFs face during greening of flashed pea leaves, has led to the development of a model of thylakoid grana in which the light-harvesting chlorophyll $a / b$ protein is envisaged as a mobile component of EFs particles $(2,3,23)$. Particle size histograms of the EFs particles from barley $(14,19)$ and maize (15) thylakoids are not characterised by sub-populations, and this can be partly explained by their asymmetrical shape $(19,20)$. A population of uniformly sized elliptical particles, 
subjected to uni-directional shadowing, does not yield a bi-modal size distribution (see 5); the values are broadly distributed about a single peak whose value is the average of the large and small axes.

Rotary shadowing was used to obtain a more accurate measure of the size and shape of the particles on fracture faces in order to overcome the decreased resolution of size distribution histograms of non-circular uni-directionally shadowed particles. The existence of discrete subpopulations of particles, distinct in shape or size, in rotary shadowed replicas could not be demonstrated for any of the fracture faces of barley thylakoids. Particle size was smoothly distributed from small to large, and shape varied in a similar manner from circular to elliptical (maximum eccentricity of 2). This raises the question of why particles exhibit such a large range of sizes and shapes, particularly those on the EFs face. These particles have been assumed to contain the reaction centres of photosystem II $(1,4)$. It has been proposed that EFs particles consist of a $80 \AA$ diameter 'core', with 1, 2 or 4 light-harvesting chlorophyll-protein complex (LHC) particles added to them $(2,3)$. Such a model explains why some particles are much larger than other EFs particles with identical function. In this model it has been assumed that the LHC component of the EFs particle projects into the intrathylakoidal space and is visible as the subunits of the multimeric ESs particles (3, 10 ), although LHC has not been demonstrated to be accessible at the inner surface of the thylakoid.

This model is inconsistant with the freezefracture ultrastructure of the chlorina- $f 2$ mutant, whose thylakoids lack LHC and all other chlorophyll $b$-containing chlorophyll proteins (9). The EFs particles of the mutant (at $126 \AA$ ) are only $12 \%$ smaller than those of wild-type ( $144 \AA$ ), whereas the above model would predict a $44 \%$ size reduction to $80 \AA$. Moreover, the persistance of tetrameric ESs particles in chlorina- $f 2$ thylakoids is not consistant with the idea that they consist of LHC. It was therefore proposed (20) that the LHC was located in particles on the PFs, most of these particles being absent in chlorina- $f 2$ thylakoids, and that the absence of chlorophyll $a / b$-protein 1 (9) might be responsible for the relatively small reduction in EFs and ESs particle size.
It is believed that ESs particles are the surface projections of EFs particles, based on the similarity of particle densities for the two faces $(19,23)$ and the formation of particle arrays of the same dimensions on both ESs and EFs (12, 13,19 , Table I). It is unexpected, therefore, that the size range of EFs particles is greater than ESs particles, and their average size smaller. Visual inspection of rotary shadowed ESs particles, particularly those in arrays $(20)$, reveals that the majority consist of four subunits and are uniformly sized. The measured size variation may be due to shadowing artifacts (18).

The larger average size of ESs particles compared with EFs particles, may be the result of plastic deformation of EFs particles during freeze-fracturing. This phenomenon is known to occur for these particles from measurements of EFs particle heights (see 20), and in model systems such as poly- $\beta$-hydroxybutyrate bodies, plastic deformation during freeze-fracturing results in a horn-like extrusion whose crosssection is both more circular and smaller than the original particle (21). In freeze-fractured LHC vesicles the electron dense particles are considered to be taller than the rest due to greater plastic deformation, and are also more circular. Plastic deformation may be responsible for the formation of smaller, more circular EFs particles, causing an increase in the range of EFs particle size, and a reduction in their average size. The question then arises whether EFs/ESs particles might not all be identical in size and shape in vivo. It is clear from this study that if sub-populations of particles exist on the EFs face, they could not be resolved by measurements from rotary shadowed replicas.

While statistically significant differences in particle size are found for the PFs face (as well as the EFs and ESs) of the chlorina- $f 2$ mutant compared with the wild-type, the major difference in the freeze-fracture ultrastructure of the mutant is that $82 \%$ of the PFs face particles are missing (Table I). It was proposed that the lightharvesting chlorophyll $a / b$-protein 2 is located in wild-type granal regions, specifically in the particles missing from the chlorina- $f 2$ face (20), which is consistent with there being no significant differences between the EFu and $\mathrm{PFu}$ faces of wild-type and mutant thylakoids (Table II). The residual particles on the chlorina- $f 2$ PFs face 
differ from those containing the light-harvesting chlorophyll $a / b$-protein 2 in both composition and size, but nevertheless are not visible as a distinct sub-population in wild-type PFs particle size measurements (Figure 1c).

In conclusion, measurements of freeze-fracture particle size and shape are complicated by several factors. Their size tends to be overestimated due to shadowing and decoration effects (18) as well as by the possibility of adsorbed layers of water (16). Particles tend to be reduced in size, particularly in their larger dimension, due to plastic deformation. On fracture faces with high particle density, mutual shading by particles will reduce the thickness of platinum deposited around each particle in rotary shadowed replicas (20), causing a further reduction in apparent particle size. These factors combine to make detailed analysis of particle size and shape difficult, and if sub-populations of differently sized particles exist, they cannot be resolved unless they differ greatly in size (probably by at least $40 \AA$ ). Nevertheless, such particle size measurements can be useful for comparing faces and in revealing differences between different fracture faces, and between corresponding fracture faces of wild-type and mutants $(14,20)$, bearing in mind the above constraints.

\section{ACKNOWLEDGEMENTS}

I am grateful to Professor Diter von WetTSTEIN for his support of this work and for helpful discussions. This work would not have been possible without the help of Jørgen Peetz, Steen Larsen and Lisbeth Sejersen, who are responsible for developing the computer programs and carrying out the statistical analyses. The skill and patience of NiNA RaSMUSSEN in drawing the figures is gratefully acknowledged.

\section{REFERENCES}

1. Armond, P. A. \& C. J. Arntzen: Localization and characterization of photosystem II in grana and stroma lamellae. Plant Physiol. 59, 398-404 (1977)

2. Armond, P. A., L. A. Staehelin \& C. J. ARNTZEN: Spatial relationship of photosystem I, photosystem II, and the light-harvesting complex in chloroplast membranes. J. Cell Biol. 73, 400418 (1977)
3. Arntzen, C. J.: Dynamic structural features of chloroplast lamellae. In: »Current Topics in Bioenergetics« (Eds. D. R. Sanadi \& L. P. Vernon). Academic Press, N.Y. Vol. VIII, $111-$ 160 (1978)

4. Arntzen, C. J., R. A. Dilley \& F. L. Crane: A comparison of chloroplast membrane surfaces visualized by freeze-etch and negative staining techniques; and ultrastructural characterization of membrane fractions obtained from digitonintreated spinach chloroplasts. J. Cell Biol. 43, 1631 (1969)

5. Carter, D. P. \& L. A. Staemelin: Proteolysis of chloroplast thylakoid membranes. II. Evidence for the involvement of the light-harvesting chlorophyll $a / b$-protein complex in thylakoid stacking and for effects of magnesium ions on photosystem II-light-harvesting complex aggregates in the absence of membrane stacking. Arch. Biochem. Biophys. 200, 374-386 (1980)

6. Fisher, K. A. \& W. Stoeckenius: Freezefractured purple membrane particles: protein content. Science 197, 72-74 (1977)

7. Giddings, T. H. JR., N. W. Withers \& L. A. StAEHELIN: Supramolecular structure of stacked and unstacked regions of the photosynthetic membranes of Prochloron sp., a prokaryote. Proc. Natl. Acad. Sci. USA 77, 352-356 (1980)

8. Goodenough, U. W. \& L. A. Staehelin: Structural differentiation of stacked and unstacked chloroplast membranes. Freeze-etch electron microscopy of wild-type and mutant strains of Chlamydomonas. J. Cell Biol. 48, 594-619 (1971)

9. Machold, O., D. J. Simpson \& B. L. Møller: Chlorophyll-proteins from wild-type and mutants of barley (Hordeum vulgare L.). Carlsberg Res. Commun. 44, 235-254 (1979)

10. McDonnel, A. \& L. A. Staehelin: Adhesion between liposomes mediated by the chlorophyll $a / b$ light-harvesting complex isolated from chloroplast membranes. J. Cell Biol. 84, 40-56 (1980)

11. Margaritis, L. H., A. Elgsaeter \& D. BranTON: Rotary replication for freeze-etching. J. Cell Biol. 72, 47-56 (1977)

12. Miller, K. R.: A particle spanning the photosynthetic membrane. J. Ultrastruct. Res. 54 159-167 (1976)

13. Miller, K. R.: Structural organization in the photosynthetic membrane. In: „Chloroplast Development" (Eds. G. Akoyunoglou et al.) Elsevier/North-Holland Biomedical Press, pp. 17-30 (1978)

14. Miller, K. R., G. J. Miller \& K. R. McIntyre: The light-harvesting chlorophyll-protein comp- 
lex of photosystem II. Its location in the photosynthetic membrane. J. Cell Biol. 7I, 624638 (1976)

15. Miller, K. R., G. J. Miller \& K. R. MCintyre: Organization of the photosynthetic membrane in maize mesophyll and bundle sheath chloroplasts. Biochim. Biophys. Acta 459, 145-156 (1977)

16. MoOR, H.: Etching and related problems. In: "Freeze-etching techniques and applications". (Eds. E. L. Benedetti \& P. Favard). Soc. Francaise Microsc. Electr., Paris, pp. 21-26 (1973)

17. Mullet, J. E., J. Burke, C. L. Ditro, J. L. Watson \& C. J. Arntzen: Integral protein complexes of chloroplast thylakoids. Plant Physiol. 63 suppl., 29 (1979)

18. Ruben, G. C. \& J. N. Telford: Dimensions of cytochrome $c$ oxidase in reconstituted liposomes using a gold ball shadow width standard: a freeze-etch electron microscope study. J. Microsc. 118, 191-216 (1980)
19. Simpson, D. J.: Freeze-fracture studies on barley plastid membranes II. Wild-type chloroplast. Carlsberg Res. Commun. 43, 365-389 (1978)

20. Simpson, D. J.: Freeze-fracture studies on barley plastid membranes III. Location of the lightharvesting chlorophyll-protein. Carlsberg Res. Commun. 44, 305-336 (1979)

21. Sleytr, U. B. \& A. W. Robards: Plastic deformation during freeze-cleavage: a review. J. Microsc. 110, 1-25 (1977)

22. Staenelin, L. A.: Analysis and critical evaluation of the information contained in freeze-etch micrographs. In: „Freeze-etching techniques and applications «. (Eds. E. L. Benedetti \& P. Favard). Soc. Francaise Microsc. Electr., Paris, pp. 113134 (1973)

23. Staehelin, L. A.: Reversible particle movements associated with unstacking and restacking of chloroplast membranes in vitro. J. Cell Biol. 71, 136-158 (1976) 